\title{
Riesgo de padecer Diabetes Mellitus Tipo 2 entre las diferentes generaciones de adultos en México
}

\section{The risk of suffering Type 2 Diabetes among different generations of adults in Mexico}

\author{
Esteban Picazzo-Palencia, Jeyle Ortiz-Rodríguez, ${ }^{*}$ \\ y Natalia Ramírez-Girón \\ Instituto de Investigaciones Sociales de la Universidad \\ Autónoma de Nuevo León, México \\ Facultad de Contaduría Pública y Administración \\ de la Universidad Autónoma de Nuevo León, México \\ Departamento de Ciencias de la Salud de la Universidad \\ de las Américas Puebla, México
}

Resumen

El presente artículo busca analizar el efecto que tiene la edad de las personas sobre la probabilidad de padecer Diabetes Mellitus Tipo 2 (DM2) entre adultos mexicanos de 20 a 59 años. Para esto se estiman ecuaciones jerárquicas con distribución Bernoulli con datos de la Encuesta Nacional de Salud y Nutrición, 2016. Los resultados indican que personas de diferentes edades presentan diferencias significativas en el riesgo de padecer DM2 independientemente de otros determinantes que pueden ser el resultado de las características del contexto y el entorno en los que crecieron. Además, se encuentra que la edad modera el efecto de tener padres con DM2 y del consumo del alcohol sobre el riesgo de padecer DM2. Los resultados indican que el mejor momento para intervenir sería en edades tempranas.

Palabras clave: Diabetes Mellitus Tipo 2; prevalencia; factores de riesgo, edad de inicio.

\section{Abstract}

This article aims to analyze the effect of age on the probability of developing Type 2 Diabetes Mellitus (2DM) among Mexican adults between 20 and 50 years old. We estimate hierarchical equations with a Bernoulli distribution using data from the National Survey of Health and Nutrition, 2016. Results indicate that individuals of different age have differences in the risk of developing 2DM. In addition, it is found that age moderates the effect of having parents with diabetes and alcohol on the risk of having diabetes. The findings reveal that the best moment to intervene is in early age.

Keywords: Type 2 Diabetes Mellitus; prevalence; risk factors, age of onset.

* Autora de correspondencia. 


\section{INTRODUCCIÓN}

nivel mundial, México ocupa la quinta posición con mayor nú1 mero de adultos diagnosticados con Diabetes Mellitus tipo 2 (DM2), siendo ésta la principal causa de muerte en el país. Se estima que 10.3 por ciento de los adultos mexicanos han sido diagnosticados con este padecimiento (Instituto Nacional de Salud Pública INSP, 2018: 6) y casi la mitad de la población se encuentra en riesgo de desarrollarla (Kumar, Wong, Ottenbacher y Snih, 2016: 164). Adicionalmente, la DM2 es el padecimiento que genera que los hogares mexicanos realicen el mayor gasto sanitario para la atención de complicaciones derivadas de ésta (Salas-Zapata y Leonardo et al., 2018: 209). Comprender las causas de la DM2 resulta esencial para el diseño de políticas públicas que mejoren el bienestar de la población. Para esto, es necesario abordar el estudio de la diabetes desde diferentes aspectos teóricos complementarios, los cuales se encuentran actualmente en la agenda de la salud pública regional (American Diabetes Association, 2018: S15). Estos temas se relacionan con cambios fisiológicos propios del aumento en la edad y el estilo de vida, que influyen directamente en la probabilidad de desarrollar la enfermedad, con determinantes ambientales y sociales que generan un efecto en las condiciones físicas individuales y repercuten en la probabilidad de padecer DM2 (World Health Organization, 2010: 53).

La relación entre los factores individuales, sociales, ambientales y el desarrollo de la enfermedad no es simple; existen controversias activas con respecto a la evidencia que respalda el papel causal de algunos factores relacionados con el contexto social, ambiental y la probabilidad de desarrollar DM2 (Braveman y Gottlieb, 2014: 25; Cockerham, Hamby, y Oates, 2017: S9). No obstante, las diferencias en los contextos y estilos de vida de las personas han sido señaladas como importantes determinantes del padecimiento de DM2 (American Diabetes Association, 2018: S15). Esto es, debido a que las personas nacidas en diferentes años experimentan contextos y estilos de vida distintos a lo largo de su ciclo de vida, podría esperarse que la probabilidad de desarrollar DM2 varíe entre personas de diferentes edades. De esta manera, el impacto de los determinantes de padecer DM2 puede depender, en gran medida, de la edad de las personas, la cual, refleja las diferencias en los contextos y expectativas de vida de cada generación. El presente artículo tiene como objetivo examinar el efecto de la edad sobre los determinantes de padecer DM2 entre los adultos mexicanos de 20 a 59 años. Para esto, se utilizan datos de la Encuesta Nacional 
de Salud y Nutrición 2016, recabada por el Instituto Nacional de Salud Pública y la Secretaría de Salud.

Utilizando un modelo jerárquico con distribución Bernoulli, en el presente estudio se busca probar tres hipótesis: i) los hábitos y las características personales tienen únicamente un efecto directo sobre la probabilidad de padecer DM2; ii) debido a que los entornos y cambios experimentados en la sociedad son diferentes para cada generación, y porque las personas envejecen de manera distinta como consecuencia de dichos cambios, la probabilidad de padecer DM2 será diferente dependiendo del año que nació cada persona; y iii) aunque las personas de mayor edad tienen mayor riesgo de padecer DM2, personas de la misma cohorte generacional poseen hábitos y características distintas que influyen de manera diferente en el riesgo de padecer DM2. Adicionalmente, existe un efecto de interacción entre los cambios experimentados en la sociedad, la predisposición genética, el desgaste fisiológico, hábitos y características personales, que genera diferencias en el riesgo de padecer DM2 entre la población.

\section{ANTECEDENTES TEÓRICOS Y EMPÍRICOS}

\section{Influencia de las características personales y hábitos}

La probabilidad de desarrollar DM2 se encuentra directamente influenciada por las características genéticas, conductas y hábitos de cada individuo. Individuos con predisposición genética o historia familiar de DM2 (familiares de primera y segunda línea de consanguinidad), tienen tejidos resistentes a la insulina, lo que facilita la hiperglucemia basal y pospandrial (Defronzo; 2009: 778). La historia familiar de DM2, explica hasta 50 por ciento la probabilidad de padecer la enfermedad, considerándose uno de los principales factores de riesgo para desarrollarla (Kral, Becker, Yanek, Vaidya, Mathias, Becker, 2019: 263).

Entre las conductas y hábitos de riesgo que aumentan la probabilidad de padecer DM2, se incluyen el patrón alimentario con exceso de sal, grasas y carbohidratos (American Diabetes Association, 2018: S15), la alteración en la calidad y cantidad del sueño (Anothaisintawee, Reutrakul, Van Cauter y Thakkinstian, 2015: 13), el consumo de tabaco, cigarrillo y alcohol (Pan, Wang, Talaei, Hu y Wu, 2015: 959), el estado de sobrepeso y obesidad, la falta de ejercicio físico y/o sedentarismo (American Diabetes Association, 2018: S15), la baja percepción de riesgo frente a DM2 (Kowall, Rathmann, Stang, Bongaerts, Kuss, Herder, Roden, Quante, Holle, Huth, Peters y Meisinger, 2017: 1) y el bajo nivel económico individual y fa- 
miliar. Por último, la evidencia señala que la presencia de Hipertensión Arterial (HTA) incrementa al doble la probabilidad de desarrollar DM2 (American Diabetes Association, 2018: S15).

\section{Diferencias en las experiencias durante el curso de vida}

La perspectiva del curso de vida considera los procesos conductuales, biológicos y psicosociales que actúan en todas las etapas de la vida: la gestación, la infancia, la adolescencia, la edad adulta y la mediana edad, como factores que pueden afectar el riesgo de desarrollar enfermedades (Baird $e t$ al., 2017: 14). Dicha perspectiva considera, en particular, la existencia de períodos "críticos" y "sensibles" a lo largo del ciclo de vida, en donde las exposiciones son deterministas o especialmente poderosas para predisponer o disminuir el riesgo de la enfermedad en el futuro (Aboderin, Kalache, Ben-Shlomo, Lynch, Yajnik, Kuh y Yach, 2002: 27).

El período crítico implica exposiciones que deben ocurrir en una o varias ventanas de tiempo específicas y, a menudo, implican exposiciones que alteran el desarrollo biológico normal. Por su parte, las exposiciones al periodo sensible se refieren a una clase más amplia de influencias que pueden tener un mayor impacto en los resultados posteriores si ocurren en ciertos períodos más que en otros. En términos empíricos, las exposiciones a periodos críticos y sensibles implican tiempo por interacciones de exposición que aumentan el riesgo de resultados adversos en salud (Baird et al., 2017: 14). En este sentido, la edad explica las diferencias en la salud y el cambio en ésta entre los diferentes grupos de edad de la población. De esta manera, los efectos de la edad por sí sola representan cambios sociales y reflejan el impacto de factores sociales exógenos en el cambio de salud en oposición a los procesos de desarrollo y envejecimiento o la progresión de la enfermedad.

Para la DM2, los cambios educativos experimentados por personas de diferentes edades, las influencias alimentarias durante los periodos de crecimiento, la apertura del mercado y exposición a productos alimentarios con alto contenido de azúcar y a sustancias psicoactivas (alcohol y cigarrillo especialmente), la deprivación social, el cambio de las tecnologías de la información, el aumento de la urbanización, el crecimiento de las ciudades, el efecto de los medios de transporte y movilidad sobre el peso (sobrepeso/ obesidad) de las poblaciones, la economía local y regional, los cambios en los sistemas de salud que influyen sobre los criterios de diagnóstico y las modalidades de promoción de salud y prevención de enfermedad tienen un efecto en la probabilidad de desarrollar DM2 (Aboderin et al., 2002: 
Riesgo de padecer Diabetes Mellitus Tipo 2 entre las diferentes generaciones de adultos en México / E. PICAZZO PALENCIA et al.

27; Hernandez-Montoya, Soriano-Flores, Esparza-Aguilar, Benjet y Llanes-Diaz, 2019: 20; Hanson, Cooper, Aihie Sayer, Eendebak, Clough y Beard, 2016: 2147). Asimismo, las instituciones, programas de salud e información en torno a la DM2 han evolucionado a lo largo del tiempo, lo que podría generar que la población tenga un riesgo diferente a padecer DM2 dependiendo del año en el que hayan nacido.

\section{Teoría de la estratificación social}

Según el Modelo sobre Determinantes Sociales de la Salud, desarrollado por la Organización Mundial de la Salud (OMS, 2010: 1), las inequidades en salud son producto de exposiciones diferentes de ciertas categorías sociales que promueven vulnerabilidades, tal como lo indica la teoría de la estratificación social, la cual es un componente esencial del Modelo de Determinantes Sociales de la Salud. De acuerdo con la teoría de la estratificación social, existen sistemas de subordinación basados en características como la raza, el origen étnico, el género y la edad; estos atributos son parcialmente moderados por el estatus socioeconómico alcanzado (educación, ingresos, estado civil), lo cual crea un dualismo en las estructuras sociales establecidas. Este dualismo provoca que la distribución de riesgos y recursos sea desigual, lo que puede originar las inequidades en salud (WHO, 2010: 1). Como los recursos de las personas pertenecientes a los grupos menos aventajados, por lo general, son limitados en comparación con el resto de la población, se genera una mala condición de salud en los grupos de personas con mayores desventajas sociales y un incremento en la brecha de inequidades en salud (Ndao-Brumblay, 2015: 36).

Las inequidades en salud en la raza, el origen étnico, el género y la edad tienen fuerte relación con el desarrollo de DM2. La raza negra y personas hispanas tienen mayor probabilidad que la raza blanca de desarrollar DM2, dado el aumento de los riesgos en los entornos socioeconómicos, psicosociales y conductuales (Piccolo, Subramanian, Pearce, Florez y McKinlay, 2016: 1217), como la baja asistencia a visitas médicas preventivas y periódicas (Taylor, Spencer, Mahabaleshwarkar y Ludden, 2019: 249), el aumento de las características comunitarias de riesgo para DM2 (Piccolo, Duncan, Pearce y McKinlay, 2015: 80), la falta de educación (Canedo, Mi1ler, Schlundt, Fadden y Sanderson, 2018: 9), el bajo nivel de autocuidado y adherencia médica (Mayberry, Bergner, Chakkalakal, Elasy y Osborn, 2016: 113).

Respecto al género, la literatura señala que las mujeres presentan mayor riesgo de DM2 debido al menor acceso a la educación, tener un ingre- 
so socioeconómico menor, mayor predisposición a alteraciones del sueño y conductas alimentarias poco saludables debido a los estilos de vida, el aumento del estrés psicosocial y laboral, asimismo al impacto de las hormonas sexuales en el metabolismo, la composición corporal, la función vascular y las respuestas inflamatorias, el incremento del riesgo de eventos cardiovasculares, mayor predisposición a la intolerancia a la glucosa y diabetes gestacional (Kautzky-Willer, Harreiter y Pacini, 2016: 280).

Con relación al impacto de la edad en el desarrollo de la DM2, éste se encuentra determinado parcialmente por las conductas de los individuos en sus estilos de vida y por las enseñanzas que toma de sus entornos; asimismo, a medida que la edad avanza, esto juega un papel importante en la insuficiencia progresiva de las células $\beta$ pancreáticas que caracteriza a la DM2 y la dificultad para el control metabólico en personas diagnosticadas con dicha enfermedad (Defronzo, 2009: 778; Mundo et al., 2019: 870).

Así, con base en la teoría de la estratificación social, el transcurso del deterioro de la salud de las personas, que evoluciona conforme se incrementa la edad, se encuentra estratificado por los hábitos y características socioeconómicas, es decir por los determinantes sociales de la salud. La estratificación social de la edad y la salud hacen referencia a las diferencias en los estados de salud entre los grupos de la población pertenecientes a diferentes grupos de edad. La causa de la relación de la estratificación del detrimento de la salud y la edad se explica en parte por las diferencias de exposición a los factores psicosociales entre los grupos poblacionales de distintas edades y con diferentes características socioeconómicas. Por otra parte, las diferencias en el impacto de los factores de riesgo de las personas de diferentes edades se encuentran determinadas por la interacción de aspectos sociales, económicos, ambientales, psicológicos, biológicos y genéticos (Shaw, Liang, Krause, Gallant y McGeever, 2010: 758).

\section{MATERIAL Y Método}

\section{Datos}

Los datos utilizados para este estudio provienen de la Encuesta Nacional de Salud y Nutrición (ENSANUT 2016) recabada por el Instituto Nacional de Salud Pública y la Secretaría de Salud (INSP, 2016: 6). La ENSANUT es una encuesta con representatividad regional, urbana, rural y nacional, y con un muestreo probabilístico, polietápico, estratificado y por conglomerados. La ENSANUT encuestó a integrantes de 9,474 viviendas; la unidad de observación fue el individuo y se seleccionaron al azar 29,795 indivi- 
duos de grupos de edad escolar (5-11 años), adolescentes (12- 19 años) y adultos (20 o más años). Las áreas temáticas incluyeron: el estado de salud y nutrición (mediciones antropométricas, análisis de sangre, historia de enfermedades crónicas y atención en el sistema de salud), acceso a servicios de salud e información socioeconómica de las personas y de la vivienda. La población objetivo de este estudio comprende a adultos de 20 años y más y el periodo de levantamiento fue entre mayo y octubre de 2016. Los datos totales de dicho grupo en la base de datos son 6,166 casos. Después de eliminar los valores perdidos y atípicos para evitar sesgos en las estimaciones, se obtuvieron un total de 6,027 casos válidos.

\section{Variables}

Diabetes. Esta es la variable dependiente del modelo, en donde se le pregunta a la persona si ha sido diagnosticada con diabetes. Se codificó como 1 si respondió de manera afirmativa y 0 de otra forma.

$E d a d$. Esta es una variable discreta que indica la edad de la persona y se utilizó para agrupar los respondientes en cohortes en el segundo nivel.

Sexo. Esta es una variable dicotómica que toma el valor de 1 si el sexo del respondiente es hombre y 0 si es mujer.

Escolaridad. Esta es una variable discreta que indica los años de escolaridad de cada persona. Esta variable se construyó con base en el número de años necesarios para obtener cada grado más los años de escolaridad del grado siguiente.

$I M C$. Esta variable indica el índice de masa corporal de los respondientes. Padres con diabetes. Esta variable toma el valor de 0 si ninguno de los padres de los respondientes fue diagnosticado con DM2, 1 si uno de los padres fue diagnosticado con DM2, y 2 si ambos padres fueron diagnosticados con DM2.

Fumar. Esta variable es discreta y toma el valor de 1 si la persona fuma actualmente, y 0 de otra forma.

Alcohol. Esta variable indica el número de veces que en los últimos 30 días la persona consumió cinco copas o más.

Hipertensión Arterial (HTA). Esta variable toma el valor de 1 si la persona ha sido diagnosticada con hipertensión, y 0 de otra forma.

Sueño. Esta variable continua indica el número de horas al día que duerme la persona.

Ejercicio. Esta es una variable continua que indica el número de horas de actividad física realizada por día. 
Percepción de Alimentación. Esta es una variable dicotómica que indica 1 si la persona encuestada considera que su alimentación es saludable, y 0 de otra forma.

\section{Modelo}

Para determinar el efecto de la edad sobre la probabilidad de padecer DM2 se estimaron las siguientes ecuaciones jerárquicas con distribución Bernoulli en el programa estadístico HLM 7.03.

Modelo nivel 1:

en donde:

$\mathrm{Y}_{\mathrm{ij}}=1$ si la persona $i$ de edad $j$ padece DM2; 0 de otra forma.

$$
\begin{aligned}
\operatorname{Prob}\left(Y_{i j}=1 \mid \beta_{j}\right) & =\frac{e^{\eta_{i j}}}{1+e^{\eta_{i j}}} \\
\eta_{i j} & =\beta_{o j}+\beta_{k j} X_{i j}+r_{i j}
\end{aligned}
$$

$\mathrm{X}_{\mathrm{ij}}=$ valores de los predictores del nivel 1 para la persona $i$ de edad $j$ (sexo, escolaridad, IMC, alcohol, hipertensión arterial, sueño, ejercicio y percepción de la alimentación).

$\mathrm{r}_{\mathrm{ij}}=$ error aleatorio asociado con la persona $i$ de edad $j$.

Modelo nivel 2:

en donde:

$\beta_{\mathrm{oj}}=$ intercepto para las personas de edad $j$.

$$
\begin{aligned}
& \beta_{0 j}=\gamma_{00}+ \gamma_{01} G_{j}+\gamma_{02} G_{j}^{2}+U_{0 j} \\
& \beta_{k j}=\gamma_{k 0}+\gamma_{k 1} G_{j}+\gamma_{k 2} G_{j}^{2}+U_{k j}
\end{aligned}
$$

$\beta_{\mathrm{kj}}=$ pendiente del predictor $k$ para las personas de edad $j$.

$\mathrm{G}_{\mathrm{j}}=\operatorname{edad} j$.

H1: Influencia de los hábitos y características personales. Las características de las personas explican cambios en la probabilidad de padecer DM2 o no y la edad no tendrá efecto sobre dichos determinantes. Diferencias en los hábitos o características relacionadas con la DM2 explican las diferencias entre las personas en la probabilidad de sufrir el padecimiento o no. Esto implica que la diferencia en la probabilidad de desarrollar DM2 
Riesgo de padecer Diabetes Mellitus Tipo 2 entre las diferentes generaciones de adultos en México / E. PICAZZO PALENCIA et al.

o no entre grupos con hábitos o características relacionadas con la DM2 se mantiene en el tiempo.

$$
\gamma_{01}=\gamma_{02}=\gamma_{\mathrm{k} 1}=\gamma_{\mathrm{k} 2}=0
$$

H2: Diferencias en las experiencias durante el curso de vida. Debido a que las personas envejecen de manera distinta porque la sociedad permanece en cambio continuo y los cambios experimentados por cada generación son distintos, la edad de las personas influye en los interceptos relacionados con el riesgo de padecer DM2. Esta hipótesis implica que personas de diferentes edades tienen diferencias en la probabilidad de tener DM2, las cuales pueden ser relacionadas con el contexto y circunstancias cambiantes para cada generación, lo que ocasiona que personas de determinadas edades sean más propensas a desarrollar DM2 sin importar la magnitud de los hábitos o características relacionadas con el riesgo de padecerla.

$$
\gamma_{01} \neq 0 \quad \text { y } \quad \gamma_{02} \neq 0
$$

H3: Teoría de la estratificación social. Las pendientes que tienen los determinantes de las personas varían entre personas de diferentes edades. El riesgo de personas de algunas edades es más sensible al efecto de los hábitos y las características personales. Es decir, la edad incrementará la brecha en la probabilidad de padecer DM2 entre diferentes grupos generacionales que adoptan hábitos no saludables y/o cuentan con características relacionadas con el incremento en la probabilidad de padecerla y el resto de los grupos. Esto implica que existe una interacción entre los cambios sociales experimentados por cada generación, la intensidad de los hábitos y características personales, la predisposición genética y el desgaste fisiológico, la cual tiene un efecto sobre el riesgo de padecer DM2.

$$
\begin{array}{rrrr}
\gamma_{\mathrm{k} 1}>0 & \text { y } & \gamma_{\mathrm{k} 2}<0 \\
& \text { o } & \\
& & \\
\gamma_{\mathrm{k} 1}<0 & \text { y } & \gamma_{\mathrm{k} 2}>0
\end{array}
$$

El análisis de los datos se realizó en el programa estadístico HLM 7.03.

\section{Análisis de resultados}

En la Tabla 1 se presentan las estadísticas descriptivas de las variables utilizadas en el modelo. En promedio, las personas de la encuesta tienen ocho años de escolaridad, 39 años, un índice de masa corporal (IMC) de 28.9 
y duermen poco más de siete horas al día. En 63 por ciento de los casos, ninguno de los padres ha sido diagnosticado con DM2, en 31.1 por ciento sólo el padre o la madre tuvo diagnóstico de diabetes y en 5.9 por ciento ambos padres tienen o tuvieron diabetes. Además, 18.3 por ciento de los respondientes padece hipertensión y 63.5 por ciento considera que tiene una alimentación saludable.

Tabla 1: Estadísticas descriptivas

\begin{tabular}{|c|c|c|c|}
\hline Variable & Media & Error estándar & \\
\hline Escolaridad & 8.15 & 4.07 & \\
\hline IMC & 28.86 & 6.80 & \\
\hline Edad & 38.82 & 10.91 & \\
\hline Alcohol & 0.79 & 1.88 & \\
\hline Sueño & 7.26 & 1.29 & \\
\hline \multirow[t]{2}{*}{ Ejercicio } & 249.70 & 218.07 & \\
\hline & Sí & No & \\
\hline Hipertensión & $18.30 \%$ & $81.70 \%$ & \\
\hline \multirow[t]{2}{*}{ Alimentación } & $63.51 \%$ & $36.49 \%$ & \\
\hline & Mujer & Hombre & \\
\hline \multirow[t]{2}{*}{ Sexo } & $67.50 \%$ & $32.50 \%$ & \\
\hline & $\begin{array}{l}\text { Sin padres } \\
\text { con diabetes }\end{array}$ & $\begin{array}{l}\text { El padre o } \\
\text { madre con diabetes }\end{array}$ & $\begin{array}{l}\text { Ambos padres } \\
\text { con diabetes }\end{array}$ \\
\hline Padres con Diabetes & $62.95 \%$ & $31.17 \%$ & $5.88 \%$ \\
\hline
\end{tabular}

Fuente: elaboración propia con datos de la ENSANUT, 2016.

Debido a que la variable dependiente es dicotómica, la varianza en el nivel 1 del modelo es heterocedástica y, por lo tanto, el índice de correlación entre clases (ICC) no revela el porcentaje de varianza explicada por la edad de las personas incluida como variable en el nivel 2 (Raudenbush y Bryck, 2002: 150). Por lo cual, como primer paso se estimó un modelo de regresión logística en donde la variable dependiente indica si la persona tiene DM2 o no y como variable independiente se utilizó la edad con el objetivo de comprobar la significancia del componente de la varianza aleatoria en el nivel 2 del modelo multinivel. Los resultados del modelo de regresión logística indican que la edad de las personas afecta de manera significativa la probabilidad de padecer DM2. Una vez que se comprobó 
que la edad tiene un efecto estadísticamente significativo en la probabilidad de padecer DM2, se procedió a estimar el modelo multinivel de las ecuaciones 1 y 2 basado en una distribución Bernoulli.

En la Tabla 2 se muestran los coeficientes del modelo de interceptos y pendientes como resultado. El modelo jerárquico planteado permite identificar el efecto moderador de la edad en las variables de primer nivel, es decir, no existe un supuesto de independencia de la probabilidad de tener DM2 entre personas de la misma edad. Las interacciones y efectos directos que no fueron estadísticamente significativos $(p>0.05)$ se eliminaron y el modelo se estimó de nuevo. El efecto directo de sexo, fumar, las horas de sueño y de actividad física no resultó significativo. Asimismo, los efectos de la edad en el segundo nivel no fueron estadísticamente significativos para el caso de las variables relacionadas con la percepción de alimentación saludable, escolaridad, IMC e HTA. Por lo cual, dichas variables y efectos se eliminaron y el modelo se volvió a estimar.

De acuerdo con los resultados, tener una alimentación saludable $\left(\gamma_{10}=-0.2208, \mathrm{p}<0.05\right)$ y mayores niveles de escolaridad $\left(\gamma_{20}=-0.0447\right.$, $\mathrm{p}<0.001)$ se encuentran relacionados de manera negativa con la probabilidad de padecer DM2. De manera contraria, los resultados indican que personas con mayor IMC $\left(\gamma_{30}=0.0178, \mathrm{p}<0.05\right)$, padres que padecieron DM2 $\left(\gamma_{40}=0.0178, \mathrm{p}<0.001\right)$, personas que consumen alcohol $\left(\gamma_{50}=0.0312\right.$, $\mathrm{p}<0.01)$ y con HTA $\left(\gamma_{60}=0.8582, \mathrm{p}<0.001\right)$ tienen mayor probabilidad de desarrollar DM2.

Con relación a los efectos de interacción, la edad modera únicamente el intercepto $\left(\gamma_{01}=0.0152, \mathrm{p}<0.01\right)$ y los efectos directos de tener padres con diabetes $\left(\gamma_{41}=0.0183, \mathrm{p}<0.05\right)$ y del consumo del alcohol $\left(\gamma_{51}=0.0100\right.$, $\mathrm{p}<0.05)$. En los tres casos, la variable edad al cuadrado se encontró estadísticamente significativa $\left(\gamma_{02}=0.0006, \mathrm{p}<0.05 ; \gamma_{42}=-0.0002, \mathrm{p}<0.05\right.$; $\left.\gamma_{52}=-0.001, \mathrm{p}<0.05\right)$. En la Figura 1 se ilustra el efecto de dichas variables en la probabilidad de padecer DM2. 
Tabla 2: Coeficientes relacionados con la probabilidad de padecer diabetes (modelo de los interceptos y pendientes como resultado)

\begin{tabular}{|c|c|c|c|c|}
\hline Efecto fijo & Coeficiente & $\begin{array}{r}\text { Error } \\
\text { estándar }\end{array}$ & $t$-ratio & $p$-value \\
\hline \multicolumn{5}{|l|}{ Intercepto, $\beta_{0}$} \\
\hline Intercepto, $\gamma_{00}$ & -3.3513 & 0.1183 & -28.334 & $<0.001$ \\
\hline Edad, $\gamma_{01}$ & 0.0152 & 0.0043 & 3.516 & 0.001 \\
\hline $\operatorname{Edad}^{2}, \gamma_{02}$ & 0.0006 & 0.0003 & 2.077 & 0.045 \\
\hline \multicolumn{5}{|c|}{ Alimentación, $\beta_{1}$} \\
\hline Intercepto, $\gamma_{10}$ & -0.2208 & 0.1090 & -2.025 & 0.043 \\
\hline \multicolumn{5}{|l|}{ Escolaridad, $\beta_{2}$} \\
\hline Intercepto, $\gamma_{20}$ & -0.0447 & 0.0132 & -3.384 & $<0.001$ \\
\hline \multicolumn{5}{|l|}{$\mathrm{IMC}, \beta_{3}$} \\
\hline Intercepto, $\gamma_{30}$ & 0.0178 & 0.0090 & 1.971 & 0.049 \\
\hline \multicolumn{5}{|c|}{ Padres con diabetes, $\beta_{4}$} \\
\hline Intercepto, $\gamma_{40}$ & 0.0178 & 0.0033 & 5.336 & $<0.001$ \\
\hline Edad, $\gamma_{41}$ & 0.0183 & 0.0092 & 1.991 & 0.047 \\
\hline $\operatorname{Edad}^{2}, \gamma_{42}$ & -0.0002 & 0.0001 & -2.115 & 0.035 \\
\hline \multicolumn{5}{|l|}{ Alcohol, $\beta_{5}$} \\
\hline Intercepto, $\gamma_{50}$ & 0.0312 & 0.0104 & 2.992 & 0.003 \\
\hline Edad, $\gamma_{51}$ & 0.0100 & 0.0047 & 2.116 & 0.034 \\
\hline $\operatorname{Edad}^{2}, \gamma_{52}$ & -0.0001 & 0.0001 & -2.007 & 0.045 \\
\hline \multicolumn{5}{|c|}{ Hipertensión arterial, $\beta_{6}$} \\
\hline Intercepto, $\gamma_{60}$ & 0.8582 & 0.1115 & 7.695 & $<0.001$ \\
\hline
\end{tabular}

Fuente: elaboración propia con datos de la ENSANUT, 2016.

Con respecto a la primera hipótesis, relacionada a la influencia de los hábitos y características personales, en donde la edad no afecta el impacto que tienen dichos determinantes, se encuentra que las variables que únicamente tienen un efecto directo sobre la probabilidad de tener DM2 son la alimentación saludable $\left(\gamma_{10}=-0.2208, \mathrm{p}<0.05\right)$, el nivel de escolaridad $\left(\gamma_{20}=-0.0447, \mathrm{p}<0.001\right)$, el IMC $\left(\gamma_{30}=0.0178, \mathrm{p}<0.05\right)$ y padecer HTA $\left(\gamma_{60}=0.8582, \mathrm{p}<0.001\right)$.

Por otra parte, la influencia de la edad y edad al cuadrado en el intercepto indica que la segunda hipótesis planteada no se rechaza. Estos resultados señalan que personas de diferentes edades tienen diferencias en la 
Riesgo de padecer Diabetes Mellitus Tipo 2 entre las diferentes generaciones de adultos en México / E. PICAZZO PALENCIA et al.

probabilidad de tener DM2 relacionada con los contextos y las circunstancias cambiantes que experimenta generación, independientemente de sus características y hábitos personales. Como se puede observar, el intercepto en la Figura 1, el efecto marginal de la edad sobre la probabilidad de tener DM2, sin considerar el resto de las variables, es creciente.

Figura 1: Efecto sobre la probabilidad de padecer diabetes, por edad
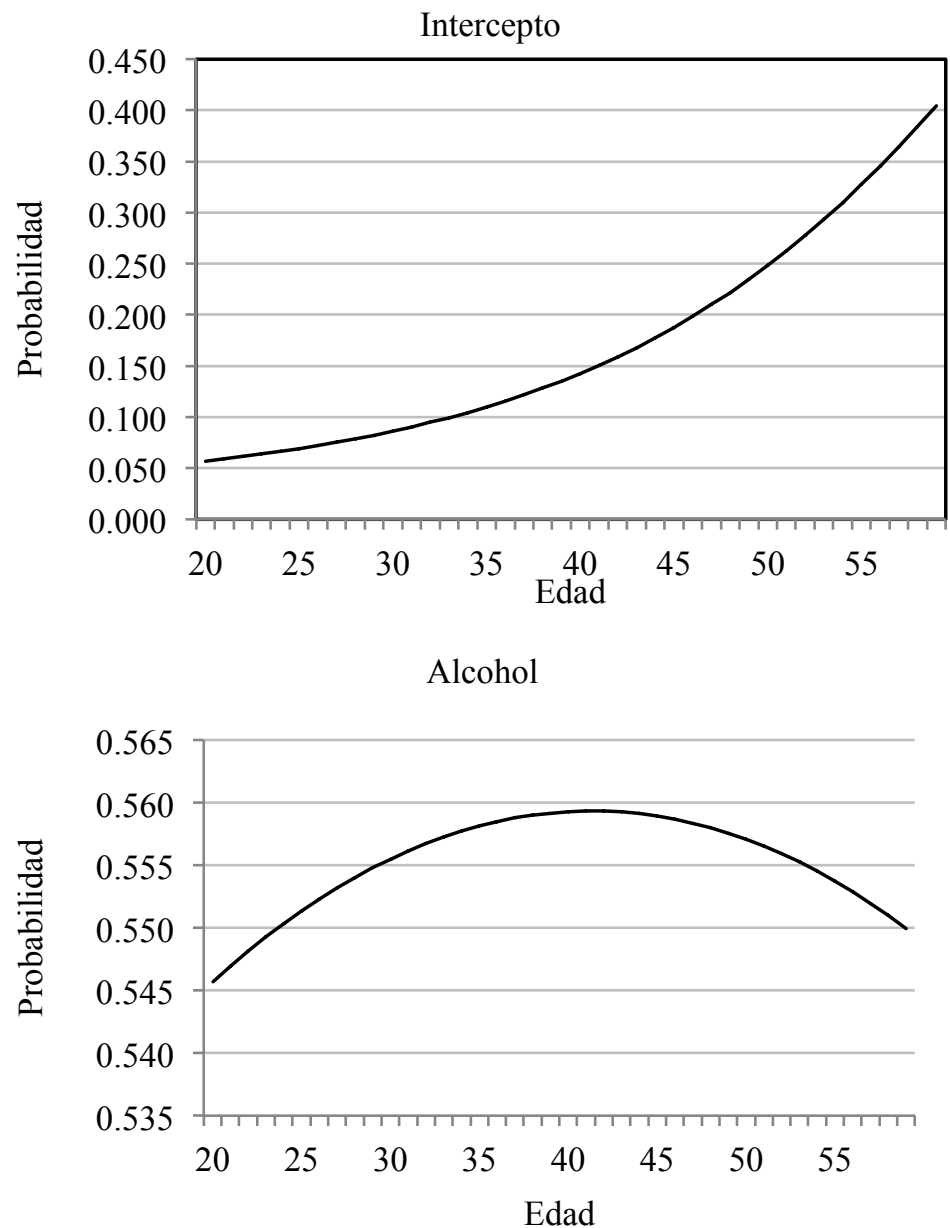

Fuente: elaboración propia con datos de la ENSANUT, 2016. 
Figura 1: Continuación

Un padre con diabetes
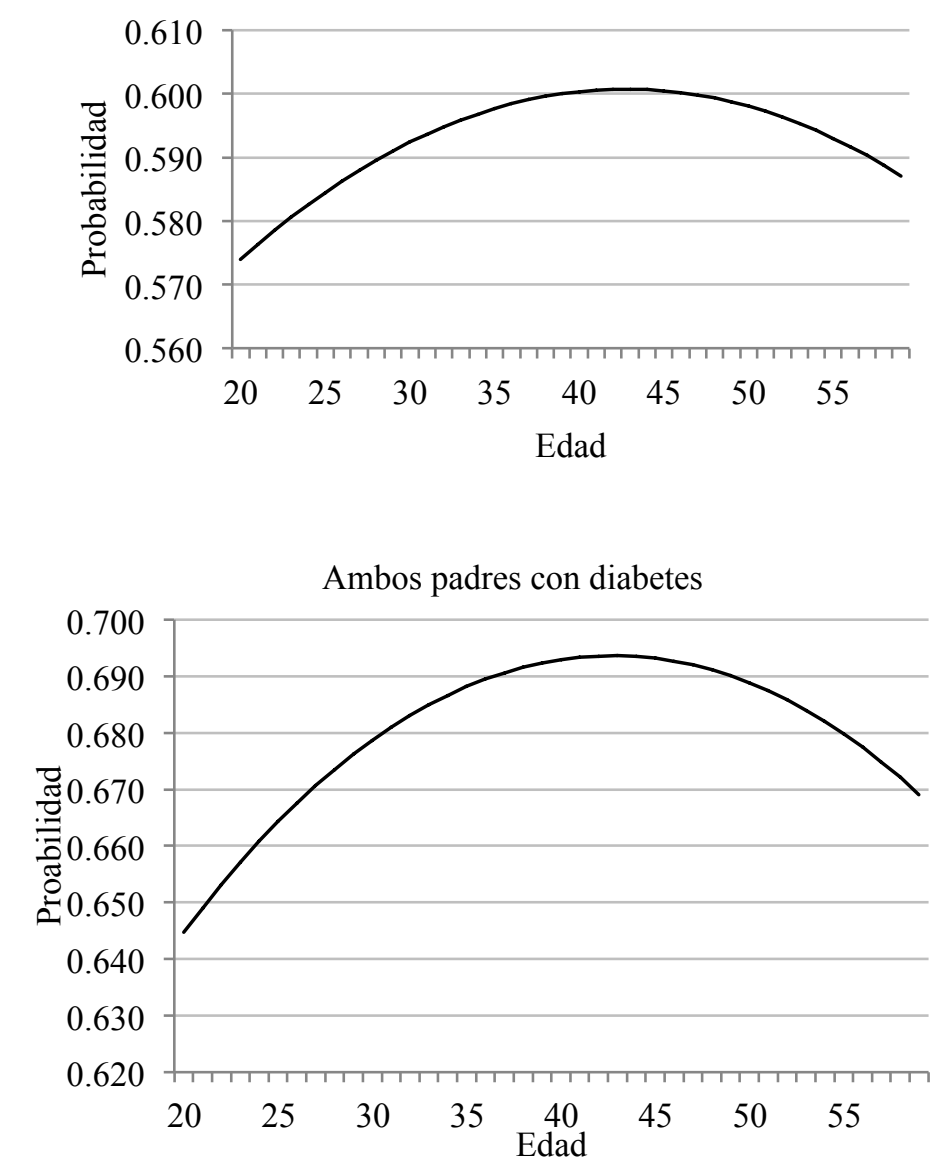

Fuente: elaboración propia con datos de la ENSANUT, 2016.

La tercera hipótesis, en la que se plantea que el efecto de los determinantes de padecer DM2 depende de la edad, no se rechaza para las variables relacionadas con el consumo del alcohol y tener o haber tenido padres con diabetes; en la Figura 1, se puede observar que ambas variables tienen un efecto sobre la probabilidad de padecer DM2 con una forma de U invertida. Respecto a los antecedentes genéticos, tener un padre diabético incrementa la probabilidad de padecer DM2 cerca de 0.60 y tener ambos padres diabéticos aumenta dicha probabilidad a alrededor de 0.70 ; en am- 
Riesgo de padecer Diabetes Mellitus Tipo 2 entre las diferentes generaciones de adultos en México / E. PICAZZO PALENCIA et al.

bos casos, la probabilidad es creciente y después comienza a disminuir alcanzando su máximo en las personas entre 37 y 50 años. De igual manera, el consumo de alcohol incrementa la probabilidad de padecer DM2 de toda la población, el efecto del consumo del alcohol tiene un incremento mayor entre personas de mediana edad (entre 35 y 48 años), en comparación con el resto de las generaciones. El consumo del alcohol tiene el mayor impacto sobre la probabilidad de padecer DM2 en personas de 41 años.

\section{DISCUSIÓN Y CONCLUSIONES}

En el presente artículo se intentó probar tres hipótesis con respecto al efecto de la edad sobre la probabilidad de padecer DM2 utilizando datos de la Encuesta Nacional de Salud y Nutrición, 2016, una muestra representativa de adultos mexicanos de 20 a 59 años. Con base en los resultados del presente estudio, se desprenden tres principales conclusiones. Primero, se encontró que tener una alimentación saludable, mayor nivel de escolaridad, el IMC y tener HTA tienen una relación directa con el riesgo de padecer DM2, y la edad no afecta el impacto que estos hábitos y características personales tienen sobre dicho riesgo. Segundo, los resultados indican que personas de diferentes edades tienen diferencias en el riesgo de padecer DM2 que pueden relacionarse como resultado del cambio en las características de los determinantes sociales intermedios de la salud en el que crecieron tales como interactuar con diferentes instituciones, información, programas y políticas públicas en torno a la prevención de la DM2. Tercero, se encontró que la edad modera el efecto de tener padres con DM2 y del consumo del alcohol sobre el riesgo de padecer DM2.

Con respecto a la primera hipótesis de este estudio en torno a la influencia de hábitos y características personales que determinan el cambio en la probabilidad de desarrollar DM2 (H1), los resultados coinciden con la evidencia sólida respecto a factores de riesgo tradicionales para DM2. Con relación a esto, en la literatura ha sido ampliamente documentado que una persona con un patrón de alimentación inadecuado, aunado a poca o ninguna actividad física probablemente tendrá un aumento en el IMC, situación que favorece la aparición de DM2 en la población (American Diabetes Association, 2018: S15; Defronzo; 2009: 778). Adicionalmente, el nivel educativo se asocia con el riesgo de DM2; diversos estudios han mostrado que, a menor nivel educativo, el riesgo e incidencia de DM2 se incrementa (Chang, J, Guy, MC., Rosales, C, de Zapien, JG., Staten, LK., Fernandez ML. et al., 2013: 3218). De esta manera, mayores niveles de escolaridad favorecen la adopción de comportamientos saludables que disminuyen la 
probabilidad de desarrollar la enfermedad (Simmons, Wolever, Bechard y Snyderman, 2014: 18). Por último, la HTA es una comorbilidad de aparición frecuente con la DM2. De acuerdo con la evidencia, alrededor de 50 por ciento de personas con DM2 padecen HTA, ambas enfermedades están inmersas en las estructuras sociales del comportamiento de la población y tiene una alta correlación entre ellas (American Diabetes Association, 2018: S15).

Con respecto a la segunda hipótesis, relacionada únicamente con el efecto de la edad y sin considerar el resto de los determinantes, los resultados de este estudio muestran que el incremento en el riesgo de padecer DM2 es creciente a medida que la edad va en aumento, es decir, el efecto de la edad tiene una forma cóncava en la probabilidad de desarrollar DM2. Estos resultados complementan la relación positiva entre la probabilidad de padecer DM2 y la edad encontrada en la literatura (Defronzo; 2009: 778). Estos hallazgos implican que la vulnerabilidad de los adultos mayores se incrementa a través del tiempo debido a la inactividad física, las comorbilidades propias del proceso de envejecimiento, el menor acceso a servicios de salud especializados y la menor generación de ingresos con relación a las personas más jóvenes (Kalyani, Golden y Cefalu, 2017: 441), así como, la brecha en las condiciones de salud y los aspectos cambiantes del contexto que están relacionados con el aumento de DM2. Entre las influencias contextuales más importantes se destaca la exposición a los entornos de alimentación poco saludables y obesogénicos que enfrenta la población. Dichos ambientes han emergido desde la segunda mitad del siglo XX y su exposición ha venido en aumento en los países de ingresos medios y bajos como efecto de la globalización, y conforme se incrementa la edad de las personas, su exposición ha venido en aumento; se consideran ambientes sociales construidos y alimentarios con influencia parcial de las reglas socioculturales y el estatus socioeconómico, estos influyen de forma importante en la transformación de los sistemas alimentarios, lo que ha provocado una disminución de acceso a alimentación saludable (Martínez Espinosa, 2017: 15). Esto ha repercutido en el aumento creciente de la composición corporal y el desarrollo precoz de enfermedades no transmisibles (ENT), específicamente DM2, en personas de diferentes generaciones que han sido expuestos a dichos cambios de manera distinta pero continua (Martínez Espinosa, 2017: 15).

Además, los resultados de este artículo sustentan lo encontrado en la literatura sobre la asociación del consumo de alcohol con el riesgo de desarrollar DM2 (Li, Yu, Zhou y He, 2016: 819). Sin embargo, en referen- 
cia a la tercera hipótesis desarrollada en el presente estudio, esta relación depende de la edad. Adicional a la edad, algunos estudios sugieren que el efecto del consumo del alcohol puede estar moderado por otras características y hábitos del comportamiento social de los individuos tales como, la cantidad de bebida alcohólica consumida, el tiempo de exposición al consumo, la actividad física, el IMC, así como el sexo; dichas características en conjunto incrementan notablemente el riesgo de padecer DM2 ( $\mathrm{Li}, \mathrm{Yu}$, Zhou y He, 2016: 819).

Por su parte, en el marco de los determinantes sociales de la salud y la estratificación social, el efecto moderador de la edad con relación a tener padres con DM2 y la probabilidad de desarrollar la enfermedad, coincide con lo reportado por algunos autores (Katulanda, Ranasinghe, Jayawardena, Sheriff y Matthews, 2015: 315), quienes encuentran que las personas con antecedentes familiares de DM2 tienen mayores de probabilidades de padecer DM2 en edades más tempranas en comparación con quienes no tienen estos antecedentes; esto relacionado altamente por los hábitos alimenticios enseñados y heredados por los padres a los hijos, asimismo a la mayor exposición a algunos factores de riesgo conductuales como patrones alimentarios inadecuados, estrés, consumo de sustancias psicoactivas reportada para los descendientes de padres con DM2 en comparación con los descendientes de padres sin DM2, los cuales, reportan menor exposición a dichos factores y entornos poco saludables (Akhuemonkhan y Lazo, 2017: 130).

Aunque se tiene poco conocimiento sobre cómo la estratificación puede afectar en el largo plazo la prevalencia de la DM2, los resultados de este artículo describen hallazgos relevantes sobre cómo actualmente la estratificación social ha incrementado la brecha del riesgo de padecer DM2 entre las diferentes generaciones de adultos en México. Dado que la estructura de la sociedad y las relaciones dentro de ella han ido cambiando permanentemente a lo largo de los últimos años, esto genera un efecto directo sobre las diferentes posiciones sociales que la sociedad le asigna a cada individuo, permitiendo a su vez exposiciones cambiantes a condiciones deletéreas para la salud y, por ende, el incremento de la vulnerabilidad. Algunas condiciones actuales, producto de la estratificación social que también pueden incrementan las inequidades en salud y que, a su vez, pueden aumentar el riesgo de DM2, son: el ingreso económico, el nivel educativo y social, la ocupación, la raza, el acceso y consumo de alimentos saludables (seguridad alimentaria), la violencia, la esperanza de vida, el acceso a sistemas de salud integrales, entre otros. El rol de las condicionantes so- 
ciales para el desarrollo de DM2 es altamente preponderante, que algunos autores, cada vez con más evidencia, la catalogan, como una enfermedad social (Domínguez, 2013: 200).

Cabe señalar que los efectos de dicha estratificación pueden variar en el tiempo, principalmente como resultado de los diferentes contextos con los que interactúa cada generación. Conforme las generaciones más jóvenes, quienes han tenido un mayor acceso a servicios de salud y más información con respecto a la DM2, reemplacen a los adultos mayores, se esperaría que las diferencias en la probabilidad de padecer DM2 sean únicamente como consecuencia del desgaste fisiológico. De esta manera, la evidencia encontrada en este estudio sobre las diferencias en el riesgo de DM2 entre adultos indica que el mejor momento para intervenir sería en las edades tempranas, dichas intervenciones deberían tener un sustento tanto fisiológico como social, dado que ambas condiciones participan en la génesis del padecimiento; en el aspecto fisiológico deberían abordarse todas las condiciones biológicas que podrían llegar a tener alteraciones patológicas y en el aspecto social, se abordaría la exposición a aspectos de los determinantes sociales de la salud potencialmente iatrogénicos para ésta; de modo que puedan llegar a ser intervenciones multicomponentes (educación, promoción, empoderamiento, entre otros) en las que se intervenga desde diversos ámbitos para la prevención de la enfermedad.

Es más probable que las personas más jóvenes cambien sus actitudes en comparación con las personas de mayor edad (Inglehart, 1990: 139). No obstante, los resultados de este estudio encuentran que la población actualmente en edad entre 35 y 50 años ya se encuentra más propensa a desarrollar DM2 como consecuencia de la estratificación. Por lo cual, podría esperarse que cuando este grupo alcance su vejez, su riesgo aumente considerablemente o sea inminente el diagnóstico de DM2, como consecuencia de hábitos adoptados de forma previa, en el marco de sus contextos vividos. En este sentido, las implicaciones de este artículo incluyen estrategias de prevención a dicho grupo de la población, con el objetivo de reducir su consumo de alcohol, así como la detección temprana de DM2. La evidencia señala que la detección temprana de la DM2 tiene un impacto significativo en el nivel del bienestar físico y mental de las personas, así como en el ámbito social para evitar los riesgos de una epidemia (Ndao-Brumblay, 2015: 36) que actualmente estamos viviendo, como lo es DM2.

Cabe señalar que el presente estudio utiliza datos representativos a nivel nacional, los cuales, permiten la generalización para la población mexicana entre 20 a 59 años, adicionalmente, se realizó un análisis que tiene 
la potencialidad de incluir diferentes niveles de anidación de los datos, los cuales pueden identificar efectos adicionales en comparación con datos sin niveles de anidación. Por otro lado, se encuentran algunas limitaciones, las cuales están relacionadas principalmente con los datos: la variable dependiente utilizada mide si la personada ha sido diagnosticada o no con DM2; no obstante, en México gran parte de las personas que padecen DM2 aún no han sido diagnosticadas. Se estima que en el país la cifra de personas que padecen DM2 podría ser del doble de las personas que han sido diagnosticadas (Instituto Nacional de Salud Pública INSP, 2016: 6). Esto podría cambiar la magnitud y nivel de significancia de los efectos encontrados en esta investigación. Adicionalmente, debido a la falta de datos tipo panel no es posible observar si las diferencias analizadas en los impactos entre cada una de las generaciones han variado a lo largo del tiempo. En este sentido, un reto para futuras investigaciones es analizar si dichas diferencias se mantienen o cambian en el tiempo.

\section{REFERENCIAS BIBLIOGRÁFICAS}

Aboderin, I., Kalache, A., Ben-Shlomo. Y., Lynch, JW., Yajnik. CS., Kuh, D. y Yach, D., 2002, Life Course Perspectives on Coronary Heart Disease, Stroke and Diabetes: Key Issues and Implications for Policy and Research. Geneva. World Health Organization. Disponible en https://apps.who.int/iris/bitstream/handle/10665/67174/WHO_NMH_NPH_02.1.pdf;jsessionid=F81E2C6D9E3E193B709CFFCD7CC305F 9 ? sequence $=1$. Accesado el 24 de julio de 2019.

American Diabetes Association, 2018, "Classification and Diagnosis of Diabetes: Standards of Medical Care in Diabetes-2018". Diabetes Care. 41(Suppl 1):S13-s27. Disponible en https://care.diabetesjournals.org/content/41/Supplement_1/S13. Accesado el 5 de julio de 2019.

Akhuemonkhan, E. and Lazo, M., 2017, "Association between family history of diabetes and cardiovascular disease and lifestyle risk factors in the United States population: The 2009-2012 National Health and Nutrition Examination Survey", in Prev Med., 96:129-34. Disponible en https://www.ncbi.nlm.nih.gov/pubmed/28007493. Accesado el 20 de agosto de 2019.

Anothaisintawee, T., Reutraku,1 S., Van Cauter, E. y Thakkinstian, A, 2015, "Sleep disturbances compared to traditional risk factors for diabetes development: Systematic review and meta-analysis", in Sleep Med Rev. 30:11-24(1). Disponible en https://www.ncbi.nlm.nih.gov/pubmed/26687279. Accesado el 8 de julio de 2019.

Baird, J. et al., 2017, "Developmental Origins of Health and Disease: A Lifecourse Approach to the Prevention of Non-Communicable Diseases", in Healthcare. 5(1):14. Disponible en http://dx.doi.org/10.3390/healthcare5010014. Accesado el 7 de agosto de 2021. 
Braveman, P. y Gottlieb, L., 2014, “The social determinants of health: it's time to consider the causes of the causes", in Public Health Rep. 129 Suppl 2:19-31. Disponible en https://www.ncbi.nlm.nih.gov/pubmed/24385661. Accesado el 20 de julio de 2019.

Canedo, JR., Miller, ST., Schlundt, D., Fadden, MK. y Sanderson, M., 2018, “Racial/Ethnic Disparities in Diabetes Quality of Care: the Role of Healthcare Access and Socioeconomic Status", in J Racial Ethn Health Disparities. 5(1):7-14. Disponible en https://www.ncbi.nlm.nih.gov/pubmed/28078660. Accesado el 8 de julio de 2019.

Chang, J., Guy, MC., Rosales, C., de Zapien, JG., Staten, LK. y Fernandez, ML, 2013, "Investigating Social Ecological Contributors to Diabetes within Hispanics in an Underserved U.S.-Mexico Border Community", in Int J Environ Res Public Health. 10(8): 3217-32. Disponible en https:/www.ncbi.nlm.nih.gov/pmc/ articles/PMC3774434/. Accesado el 8 de agosto de 2019.

Cockerham, WC., Hamby, BW. y Oates, GR., 2017, "The Social Determinants of Chronic Disease", in Am J Prev Med. 52(1S1):S5-S12. Disponible en https://www. ncbi.nlm.nih.gov/pmc/articles/PMC5328595/. Accesado el 19 de julio de 2019.

Defronzo, RA., 2009, "Banting Lecture. From the triumvirate to the ominous octet: a new paradigm for the treatment of type 2 diabetes mellitus", in Diabetes; 58(4):773-95. Disponible en https://www.ncbi.nlm.nih.gov/pmc/articles/ PMC2661582/. Accesado el 9 de julio de 2019.

Domínguez, Alonso Emma, 2013, "Desigualdades sociales y diabetes mellitus", in Rev Cubana Endocrinol. 24(2): 200-213. Disponible en http://scielo.sld.cu/scielo. php?script=sci_arttext\&pid=S1561-29532013000200009\&lng=es. Accesado el 9 agosto de 2021 .

Hanson, MA., Cooper, C., Aihie Sayer, A., Eendebak, RJ., Clough, GF. y Beard, JR., 2016, "Developmental aspects of a life course approach to healthy ageing", in $J$ Physiol. 594(8):2147-60. Disponible en https://www.ncbi.nlm.nih.gov/pub$\mathrm{med} / 26518329$. Accesado el 5 de julio de 2019.

Hernandez-Montoya, D., Soriano-Flores, A., Esparza-Aguilar, M., Benjet, C. y Llanes-Diaz, N., 2019, "Variation in incidence of type 2 diabetes mellitus: time series of Mexican adolescents", in Ann Epidemiol. 30:15-21. Disponible en https://www.ncbi.nlm.nih.gov/pubmed/30528324. Accesado el 21 de julio de 2019.

Inglehart, R., 1990, Culture Shift in Advanced Industrial Society. Princeton: Princeton University Press.

INSP, 2016, Encuesta Nacional de Salud y Nutrición de Medio Camino ENSANUT MC 2016. Instituto Nacional de Salud Pública, Cuernavaca, Morelos; Disponible en https://www.gob.mx/cms/uploads/attachment/file/209093/ENSANUT.pdf.Accesado el 4 julio de 2019.

INSP, 2018, Encuesta Nacional de Salud y Nutrición de Medio Camino ENSANUT 2018. Instituto Nacional de Salud Pública, Cuernavaca, Morelos. Disponible en https://ensanut.insp.mx/encuestas/ensanut2018/doctos/informes/ensanut_2018_ presentacion_resultados.pdf. Accesado el 7 agosto de 2021. 
Riesgo de padecer Diabetes Mellitus Tipo 2 entre las diferentes generaciones de adultos en México / E. PICAZZO PALENCIA et al.

Kalyani, RR., Golden, SH. y Cefalu, WT., 2017, "Diabetes and Aging: Unique Considerations and Goals of Care", in Diabetes Care. 40(4):440-443. Disponible en https:/www.ncbi.nlm.nih.gov/pmc/articles/PMC5360288/. Accesado el 15 de agosto de 2019.

Katulanda, P., Ranasinghe, P., Jayawardena, R., Sheriff, R. y Matthews, DR., 2015, "The influence of family history of diabetes on disease prevalence and associated metabolic risk factors among Sri Lankan adults", in Diabet Med. 32(3):314-23. Disponible en https://www.ncbi.nlm.nih.gov/pubmed/25251687. Accesado el 20 de agosto de 2019.

Kautzky-Willer, A., Harreiter, J. y Pacini, G., 2016, "Sex and Gender Differences in Risk, Pathophysiology and Complications of Type 2 Diabetes Mellitus", in Endocr Rev. 37(3):278-316. Disponible en https://www.ncbi.nlm.nih.gov/pub$\mathrm{med} / 27159875$. Accesado el 8 de agosto de 2019.

Kowall, B., Rathmann, W., Stang, A., Bongaerts, B., Kuss, O., Herder, C., Roden, M., Quante, A., Holle, R., Huth, C., Peters, A. y Meisinger, C., 2017, "Perceived risk of diabetes seriously underestimates actual diabetes risk: The KORA FF4 study", in PLoS One. 31;12(1):e0171152. Disponible en https://www.ncbi.nlm. nih.gov/pmc/articles/PMC5283734/. Accesado el 25 de julio de 2019.

Kral, BG., Becker, DM., Yanek, LR., Vaidya, D., Mathias, RA., Becker, LC., 2019, "The relationship of family history and risk of type 2 diabetes differs by ancestry, in Diabetes Metab. 45(3):261-7. Disponible en https://www.ncbi.nlm. nih.gov/pubmed/29875064. Accesado el 29 de julio de 2019.

Kumar, A., Wong, R., Ottenbacher, KJ. y Al Snih, S., 2016, "Prediabetes, undiagnosed diabetes, and diabetes among Mexican adults: findings from the Mexican Health and Aging Study", in Ann Epidemiol. 26(3):163-70. Disponible en https:// www.ncbi.nlm.nih.gov/pubmed/26872919. Accesado el 3 julio de 2019.

Li, XH., Yu, FF., Zhou, YH. y He, J., 2016, “Association between alcohol consumption and the risk of incident type 2 diabetes: a systematic review and dose-response meta-analysis", in Am J Clin Nutr. 103(3):818-29. Disponible en https:// www.ncbi.nlm.nih.gov/pubmed/26843157. Accesado el 20 de agosto de 2019.

Martínez Espinosa, A., 2017, "La consolidación del ambiente obesogénico en México", en Estudios Sociales. 27:50. Disponible en http://www.scielo.org.mx/ scielo.php?script=sci_arttext\&pid=S0188-45572017000200006. Accesado el 10 de agosto de 2019.

Mayberry, LS., Bergner, EM., Chakkalakal, RJ., Elasy, TA. y Osborn, CY., 2016, "Self-Care Disparities Among Adults with Type 2 Diabetes in the USA", in Curr Diab Rep. 16(11):113. Disponible en https://www.ncbi.nlm.nih.gov/pubmed/27671320. Accesado el 8 de agosto de 2019.

Mundo-Rosas, V., Unar-Munguía, M., Hernández, F., Pérez-Escamilla, R., y Shamah-Levy, T., 2019, "La seguridad alimentaria en los hogares en pobreza de México: una mirada desde el acceso, la disponibilidad y el consumo", en Salud Pública de México, 61(6), 866- 875. https://doi.org/10.21149/10579. Accesado el 7 de agosto de 2021. 
Ndao-Brumblay, SK., 2015, Health Changes after Diabetes Diagnosed in Middle and Old Age: Physical, Mental and Cognitive Health Trajectories and Social Stratification, Michigan: The University of Michigan; 2015. Disponible en https:// deepblue.lib.umich.edu/handle/2027.42/113478. Accesado el 5 de julio de 2019.

Pan, A., Wang, Y., Talaei, M., Hu, FB. and Wu, T., 2015, "Relation of active, passive, and quitting smoking with incident type 2 diabetes: a systematic review and meta-analysis", in Lancet Diabetes Endocrinol. 3(12):958-67. Disponible en https://www.ncbi.nlm.nih.gov/pubmed/26388413. Accesado el 28 de julio de 2019.

Piccolo, RS., Duncan, DT., Pearce, N. y McKinlay, JB., 2015, “The role of neighborhood characteristics in racial/ethnic disparities in type 2 diabetes: results from the Boston Area Community Health (BACH) Survey", in Soc Sci Med. 130:79-90. Disponible en https://www.ncbi.nlm.nih.gov/pubmed/25687243. Accesado el 8 de julio de 2019.

Piccolo, RS., Subramanian, SV., Pearce, N., Florez, JC. y McKinlay, JB., 2016, "Relative Contributions of Socioeconomic, Local Environmental, Psychosocial, Lifestyle/Behavioral, Biophysiological, and Ancestral Factors to Racial/Ethnic Disparities in Type 2 Diabetes", in Diabetes Care. 39(7):1208-17. Disponible en https://www.ncbi.nlm.nih.gov/pubmed/27330127. Accesado el 5 de julio de 2019.

Raudenbush, SW. y Bryk, AS., 2002, "Hierarchical linear models: Applications and data analysis methods". Thousand Oaks, CA.: Sage; 2002.

Salas-Zapata L., Palacio-Mejía, LS., Aracena-Genao, B., Hernández-Ávila, JE., Nieto-López, ES., 2018, "Costos directos de las hospitalizaciones por diabetes mellitus en el Instituto Mexicano del Seguro Social”, en Gac Sanit. 32(03):209215. Disponible en https://doi.org/10.1016/j.gaceta.2016.06.015. Accesado el 5 de agosto de 2021.

Shaw, BA., Liang, J., Krause, N., Gallant, M. y McGeever, K., 2010, “Age differences and social stratification in the long-term trajectories of leisure-time physical activity", in J Gerontol B Psychol Sci Soc Sci. 65(6):756-66. Disponible en https:// www.ncbi.nlm.nih.gov/pubmed/20855534. Accesado el 8 de agosto de 2019.

Simmons, LA., Wolever, RQ., Bechard, EM. y Snyderman, R., 2014, "Patient engagement as a risk factor in personalized health care: a systematic review of the literature on chronic disease". Genome Med. 6(2):16. Disponible en https://www. ncbi.nlm.nih.gov/pubmed/24571651. Accesado el 18 de agosto de 2019.

Taylor, YJ., Spencer, MD., Mahabaleshwarkar, R. y Ludden, T., 2019, "Racial/ethnic differences in healthcare use among patients with uncontrolled and controlled diabetes", in Ethn Health. 24(3):245-56. Disponible en https://www.ncbi.nlm.nih. gov/pubmed/28393538. Accesado el 5 de julio de 2019.

World Health Organization, 2010, "A conceptual framework for action on the social determinants of health”. Geneva: WHO; Disponible en https://www.who.int/ sdhconference/resources/ConceptualframeworkforactiononSDH_eng.pdf. Accesado el 10 de julio de 2019. 
Riesgo de padecer Diabetes Mellitus Tipo 2 entre las diferentes generaciones de adultos en México / E. PICAZZO PALENCIA et al.

\section{RESUMEN CURRICULAR DE LOS AUTORES}

\section{Esteban Picazzo Palencia}

Licenciado en Economía por la Universidad Autónoma de Nuevo León. Maestro en Economía y Doctorado en Ciencias Sociales por la Universidad Autónoma de Nuevo León. Investigador Nivel I del Sistema Nacional de Investigadores. Actualmente se desempeña como Profesor e Investigador en el Instituto de Investigaciones Sociales (IINSO) de la Universidad Autónoma de Nuevo León. Ha desarrollado trabajos de investigación en Economía de la Salud y Sustentabilidad del Desarrollo.

Dirección electrónica: epicazzo@yahoo.com

Registro ORCID: https://orcid.org/0000-0002-2456-7955

\section{Jeyle Ortiz Rodriguez}

Licenciada en Economía por la Universidad Autónoma de Nuevo León (UANL) y Maestría en Economía y Políticas Públicas por la Escuela de Graduados de Administración Pública y Política Pública. Doctorado en Filosofía con orientación en Trabajo Social y Políticas Comparadas de Bienestar Social por la Universidad de Texas en Arlington. Fue becaria Fulbright durante sus estudios de doctorado. Actualmente trabaja como Profesora-Investigadora en la Facultad de Contaduría Pública y Administración de la UANL. Ha sido Profesora Invitada en la Escuela de Verano sobre Economías Latinoamericanas de la CEPAL. Obtuvo el Premio de la Excelencia en Estudios de Mujeres y Género en 2016 por la Universidad de Texas en Arlington. Sus líneas de investigación son género y bienestar social. Es miembro del Sistema Nacional de Investigadores Nivel 1.

Autora de correspondencia.

Dirección electrónica: jeyleortiz@gmail.com

Registro ORCID: https://orcid.org/0000-0001-6220-7742

\section{Natalia Ramírez Girón}

La Dra. Natalia Ramírez Girón es originaria de Quindío, Colombia, egresó de la Licenciatura en Enfermería en la Universidad del Quindío, posteriormente culminó la Maestría en Enfermería en la Benemérita Universidad Autónoma de Puebla (BUAP) y el Doctorado en Ciencias de Enfermería en la Universidad Autónoma de Nuevo León (UANL). En su trayectoria profesional se destacó como enfermera clínica de sala de hemodiálisis. Además, tiene experiencia docente a nivel licenciatura y posgrado en escuelas públicas y privadas de Enfermería, cuenta con experiencia en inves- 
tigación, es revisora editorial de revistas nacionales e internacionales. $\mathrm{Su}$ área de estudio se ha enmarcado en el abordaje socio-ecológico y familiar del adulto en riesgo y con Diabetes Mellitus tipo 2. Actualmente, es profesora de tiempo completo del departamento de Ciencias de la Salud de la Universidad de las Américas Puebla. Es candidata del Sistema Nacional de Investigadores.

Dirección electrónica: natalia.ramirez.giron@gmail.com

Registro ORCID: https://orcid.org/0000-0002-8312-6287 